\title{
La poesia di Bodini tra ermetismo e il surrealismo di Lorca
}

Nel processo di maturazione critica cui va incontro l'opera di Vittorio Bodini-processo lento, discontinuo, distinto comunque da esiti pregevoli, testimone il recente volume Le terre di Carlo $V^{1}$ - viene ribadito, anche rispetto alla precedente antologia di scritti critici (Mancino 391), quanto abbia inciso l'esperienza spagnola sulla formazione del poeta leccese. Rilevato da tempo, ma quasi sempre in termini approssimativi, ${ }^{2}$ il contributo del surrealismo spagnolo al rinnovamento della poesia di Bodini; indivuate, da Oreste Macrí (Introduzione a Bodini 36 passim), costellazioni di immagini, stilemi e nuclei lessicali che si riportano alla generazione del Ventisette, resta comunque da approfondire il raggio e lo spessore di quell'intenso rapporto con la cultura spagnola che si rispecchia ne La Luna dei Borboni (1952) e Dopo la Luna (1956). È quanto si propone questo studio, ma solo in parte giacché si limita all'apporto di una pocta, Federico García Lorca, indubbiamente la voce piú costante nel percorso poctico di Bodini, segnato altresí dalla presenza di Rafael Alberti, Vicente Aleixandre, Luis Cernuda e Pedro Salinas. Va precisato che un confronto tra Bodini e Lorca (ma può dirsi lo stesso di altri poeti spagnoli) si giustifica anzitutto quale tentativo di maggior approfondimento critico, poiché ci troviamo di fronte a una produzione letteraria ove la disponibilità all'assimilazione, in quanto aliena all'imitazione tout court, si traduce in elezione di particolari strutture verbali, le quali si trasformano e si rinnovano nell'evoluzione di un esercizio poetico sempre teso alla ricerca di un'espressività autentica e originale.

È noto che l'accostamento di Bodini alla cultura spagnola nasce come anelito di alterazione al contatto piú o meno assiduo con l'ermetismo fiorentino (1939-40) che lascerà comunque segni ben precisi sull'opera bodiniana anche nelle sue fasi evolutive. Animato dalle prospettive di "un'altra via, un altro linguaggio poetico,", Bodini si allontana dall'ambiente delle Giubbe Rosse quando scorge nell'esperienza ermetica una condizione di stasi, restia a sollecita- 
zioni di rinnovamento che il poeta non esiterà a definire "avversione a calarsi nel fondo di sé" (Macrí, Introduzione 16). Poche e di scarso rilievo le testimonianze del periodo fiorentino, contrassegnato da un linguaggio soffuso di grigiore crepuscolare, portato alla figurazione incerta di giovanili e abulici umori esistenziali che si irrigidiscono in un"atmosfera di "pigri errori di nebbic e avvolgimenti" tali da "chiudere" l'io poetico "in un orbita" di velleitari ricordi (Giardini D'Azeglio, API ). ${ }^{4}$ Di maggior interesse le prove connesse al periodo leccese (1940-44) e quello romano (1944-46), costituite da un gruppo di circa 50 poesie quasi tutte inedite in vita di cui l'edizione curata da Macrí ci permette di ricostruirne i moventi e l'itinerario. Risultano prove di aneliti transitivi, conato di dilatazione dell'ermestismo in direzione di un linguaggio inedito che pur affiora in qualche verso di rilievo-si pensi al "saluto dei gabbiani" intravisto in "un bianco fascio / slegato di garofani” (I/ gatto eumuco, API). Da simili tentativi di rinnovamento non emerge comunque un orientamento organico né un filo conduttore. Si verifica il contrario: un registro polifonico fatto di richiami che risalgono a Foscolo (Macrí, Foscolo 129-133), al Leopardi (si veda Zibaldone leccese, API), al D’Annunzio c al Montale di Ossi di seppia c La bufera. In questa fase di ricerca e di sperimentazione "per trovare," dirà l'autore, "un linguaggio piú libero e da poter dire mio" (nota Preliminare di Bodini a LB riportata da Macrí, edizione di Bodini 90), si hanno i primi contatti con la letteratura spagnola. Attraverso "voraci letture e fitte traduzioni specialmente di poeti novecenteschi” (Macrí, Introduzione 20), Bodini scopre il fascino del surreale, di cui non mancano testimonianze esemplari: "Gli alberi si nutrivano degli occhi / di lamentosi uccelli" (Gli aiberi si mutrivano. . ., API); "Ma nell'ora in cui tutti gli orologi / si lasciano morire dietro i vetri” (Ma nell'ora. . . API). Di notevole interesse la presenza di Lorca, ravvisabile nel verso "Rose lune delirano nei prati" (Egloga, $A P I)$, nel "sangue acceso," nel "galoppo di nervosi cavalli" (Un'astrazione fervida, API) e nella Processione del Venerdi Santo (API), stillato "alla maniera di Federico García Lorca," che risulta peraltro ben lungi da quell'integrazione lirico-strutturale, precisa e vibrante, propria del periodo spagnolo.

Dalla sperimentazione priva di contenuti organici alla dimora in Spagna, che ha inizio nell'autunno del ' 46 e si protrac fino alla primavera del ' 49 anche se interrotta da un breve soggiorno in patria 
nell'estate del '47. Chi voglia confrontare la produzione intensa ma tuttora indistinta del periodo leccese-romano con le affermazioni liriche congiunte all'esperienza spagnola che faranno parte de La luna dei Borboni e altre poesie (1962) non avrà difficoltà nel constatare quanto sia rilevante "la grave svolta" nell'iter poetico bodiniano scgnalata dall'autore all'amico e conterraneo Oreste Macrí (Macrí, Introduzione 28). Allc condizioni che preparano la "svolta" di Bodini va premessa l'autocoscienza di un impasse vissuto dal poeta negli anni romani che si risolve in aperta disponibilità alle sollecitazioni del surrealismo spagnolo, orientamento, questo, aperto sí ad alcune istanze del surrealismo francese, quali l'automatismo, l'inconscio e la dimensione dell'onirico, ma volto anzitutto al rinnovamento strutturale della lirica spagnola, rinnovamento mediato dalla "rivoluzione espressiva" di Góngora, il quale, scrive Bodini, "spinse fino ad estremi insuperabili i propri mezzi tecnici” (I poeti XXVII). Integrato all'humus culturale iberico con l'apporto del conceptismo gongorino, il surrealismo della generazione del Ventisette mirava alla concentrazione del lessico, al complesso e fascinoso gioco della metafora, alla trasfigurazione del reale.

Da questi fermenti e dalle esemplifacazioni che convergono nei testi di Lorca, Salinas c altri, prende avvio in Bodini un processo di innovazione in base al quale il suo canzonicre, sfoltito di forme nebulose, eclettiche e a volte discordi, trova piena espressione nella raffigurazione mitico-emblematica del Salento. Si profila cosí uno stretto rapporto tra Lorca e Bodini, rapporto che ha come risvolti piú significativi l'autenticità, indice di approdo e rinnovamento artistico di cui il poeta leccese si rende partecipe quando matura la consapevolezza che l'atto di compenetrazione, cmotivo e intellettuale, del sostrato culturale della Spagna, e in particolare dell'Andalusia, porta alla scoperta di segni rivelatori e inconfondibili della propria terra. Spagna quindi come immagine del Sud curopeo, Andalusia il suo entroterra mitico e favoloso che nella raffigurazione magistrale di García Lorca si offre quale condizione di perenne tensione nei confronti della realtà. Se, come rileva Macrí, al centro del Romancero gitano "stanno il viaggio sotterraneo, la peregrinatio orphica alle radici di Andalusia” (Poesia LIII), possiamo senz'altro sostenere che Bodini percorre lo stesso iter poetico nelle viscere del suo Salento, riclaborando e reinventando categorie vitali di stampo lorchiano: la 
luna, il sangue, la morte, il cavallo, il vento. Spagna come metafora del Sud, e non solo del Sud europeo. "E poi, dissi, per Maldrid ho delle ragioni speciali"-scriveva Bodini nel 1951_- "sono un italiano del Sud, e questa dovrebbe essere la vera capitale del mio paese. Vi è in noi la medesima combinazione di follia e realismo, le stesse incrzic febbrili, lo stesso bianco della calce contro il cielo" ("Madrileno"). ${ }^{5}$ Dal bisogno di ridefinire la propria meridionalità in sintonia con una cultura affine nasce "il flamenco salentino" (sono parole di Macrí), la trasposizione del cante jondo in terra pugliese, il canto andaluso che Bodini tenne a definire "una cosa dell'anima" (Introduzione) e in virtú del quale si concretizza l'espressione del sentimento nero radicato nell'anima del Sud, nella coscienza del suo destino secolare di asservimento, di sofferenza e miseric:

Sulle pianure del Sud non passa un sogno.

Sostantivi e le capre senza musica, con un segno di croce sulla schiena, o un cerchio quivi accampati aspettano un'altra vita. $(3, L B)$

Bodini intuisce la necessità di un rapporto assoluto con la sua terra, e tale rapporto si effettuerà sul filo di uno scavo lessicale intenso e febbrile nel suolo arido del Salento dal quale emergono "dentature di cavalli / uccisi" $(3, L B)$ c "l'acciaio senza luce d'antiche spade" (Ibid.)-detriti di un passato preistorico intriso di sangue cui fa da contrappunto la figura del "carretticre decapitato" $(5, F T)$, immagine emblematica della realtà contemporanea delle "terre del Sud" ove "l'aria c̀ piena di sangue" $(5, F T)$. Si c̀ voluto insistere, non senza giustificazione, sulla raffigurazione di un Sud primitivo c violento quale icone di una comunità agrestre caduta in oblio i cui segni vitali il poeta riporta alla luce anche in rapporto al tentativo di ricerca della propric origini (Perricone 302-320). Eppure nella proiezione di una realtà che porta i contrassegni lorchiani del sangue e della spada si riconosce maggiormente, a nostro avviso, il sentimento offeso di un uomo che ha dietro le spalle anche la barbarie del suo secolo, la ferocia del secondo conflitto mondiale, i "gridi" inascoltati della sua terra natale.

Al nostro tentativo di porre in rilievo testimonianze piú esplicite della presenza di Lorca nella poesia di Bodini va premesso che si tratta di un rapporto poliedrico costituito da sintonic, sollecitazioni, 
evocazioni, quasi mai di ricalchi o assimilazioni dirette. Del Romancero gitano, La Luna dei Borboni riflette un intenso fervore poetico che è angoscia e passione, ne riflette la pena del vivere quotidiano l'impulso conoscitivo del logos che trae alimento dalle "radici della terra e del sangue," come ha rilievato Donato Valli (319). ${ }^{6}$ Nella strutturazione del periodo poetico non mancano strofe esemplari in cui il discorsivo di Bodini-ad esempio, "Quando seppe l'aumento del prezzo dei pomodori / capí che il tempo dei palpiti era finito" (Morta in Puglia, LB)_richiama il testo lorchiano di Poeta en Nueva York: "Cuando se hundieron la formas puras / [ . . . ] comprendí que me habían asesinado" (Fábula y rueda de los tres amigos). ${ }^{7}$ Probabile anche il contributo di Aleixandre nel confronto analogico tra "il tempo dei sospiri era finito" ed "el tiempo de los besos no ha llegado" (140). Raffronti, questi, che possono anche ammettere l'ipotesi di un ricalco formale; in effetti, risulta piú valido parlare di testimonianze, di richiami voluti sub specie di riconoscimento e di "gidiano influsso d'elezione" (Boselli 99) nei confronti della poesia spagnola. Lo stesso può dirsi del ritmo lento e penoso che scandisce l'iterativo "hai fatto bene a non parlarmi del Sud / del Sud" (Le mani del Sud, $C I)$ e dei tre versi del poema Madrid (VDA), "Lo stagno senza viole dove morí Pilar / [. . . ] non lo voglio vedere," che si riportano, per vibrazioni tonali e corrispondenze emotive, al Llanto por Ignacio Sánchez Mejías. Affinità quindi di registri espressivi sottesi da un sentimento comune di angosciose tensioni esistenziali. Si pensi all'atmosfera tesa e pregna di insidic del Romancero gitano in relazione ai seguenti versi di Calle del Pez $(L B)$, omonimo domicilio madrileno di Bodini:

Che ricamo di fiamme su un vuoto petto!

Che furia d'aghi da lontano,

e a batter a ogni porta: "Che sapete

voi del mio viaggio?"

Volendo approfondire i riscontri semantici, si potrà ricordare, sempre del Lorca, un verso giovanile che qualifica il "vuoto petto" bodiniano: "Hay en mi pecho una hondura / de sepultura" (Paisaje, LP).

Piú incisive risultano le evocazioni dal tessuto poetico lorchiano di termini-chiave che nel contesto de La luna dei Borboni assumono la funzione di vere matrici metaforiche: sangue, morte, preistoria, scheletri, teschi, oltre ovviamente alla presenza costante della luna. 
Del Sálento arido ed essenziale, del suo paesaggio che nasconde i relitti di un passato violento, la poesia di Lorcal offre echi e suggestivi richiami:

Mira el ansia, la angustia de un triste mundo fósil
que no encuentra el acento de su primer sollozo.

(Nocturno, PNY)

e

La granada es la prehistoria

de la sangre que llevamos,

la idea de la sangre. (Cancion oriental, LP)

In effetti, un confronto attento tra il Romancero gitano e La luna dei Borboni pone in evidenza la presenza di nuclei lessicali di affinità tutt'altro che casuali: "corteza de hastío": "il seme della noia"; "esquéletos del gavilanes": "spettri di capre"; "nidos de oro": "pesci d'oro"; "viento verde": "verde vento"; "dentadura de caballo": "dentature di cavalli"; "un silencio hecho pedazos": "cade a pezzi . . . un tramonto." Del "viso sfregiato" della luna dei Borboni si ha riscontro preciso nel "dolor de luna apuntillada" del poema Nocturno de hueco $(P N Y)$ ove il profilo lunare di "gesso tranquillo" assume lentamente forma muliebre dal volto freddo e malefico: "En la dura barraca. .. . la luna prisionera / devora a un marincro delante de los niños"; in Bodini troviamo la luna dai "capelli corti. . . ghiotta d'angurie" (4, $L B)$ nonché la luna che "suscita falsi monti / che paiono uccisi" (2, $F T$ ). Rilevante, anche come conferma di stilemi analogici connessi alla stessa immagine, "la mentirosa luna de los polos" (Los negros, PNY) e, di Bodini, "I'inefficace luna / schiacciata ai poli" (Tutto un paese sorge contro un uomo, $D L$ ). La luna di Lorca, definita dal poeta leccese "luna meridionale dalle ombre vicine tagliate col coltello" (I poeti LXVIII), ̀̀ sentita e raffigurata dal Nostro in termini affini, quale segno eversivo del romantico, archetipo di connivenze e corresponsabilità nel destino oscuro degli uomini.

Si è detto che il rapporto di Bodini con il surrealismo spagnolo si articola maggiormente in termini di assimilazioni elettive che passano attraverso il filtro della mediazione prima di essere integrate al tessuto poetico bodiniano. Ne risulta una scrittura che porta come contrassegni di rinnovamento la concentrazione del lessico, l'acuità dell'angoscia esistenziale, e un'intensità lirica che ha pochi riscontri 
nella poesia italiana del dopoguerra. Dal contatto assiduo con l'opera di Lorca, contatto nutrito anche dall'csercizio della traduzione, ${ }^{8}$ la lirica di Bodini risente soprattutto la tensione e la forza della metafora messa a nudo. Si pensi ad esemplificazioni lorchiane quali "Como un río de lcones / su maravillosa fuerza" (La sangre derramada, LPI); "La frenética lluvia di mis venas" (Soledad, PS); "Se agitan en mi cerebro / dos palomas campesinas" (El diamante, LP); "El niño llora / en el vientre di su madre" (San Gabriel, $R G$ ); "El sol se lleva tu alma / para hacerla luz" (Cigarra! LP) oltre a nuclei iconici come "tronco sangrante" (Nocturno del hueco, PNY), "negro toro de pena" (La sangre derramada, LPI); "un cielo talondrado de estrellas" (Elegía del silencio, LP); "un torrente de luceros" (Manatial, $P N Y$ ); "naufragio de sangre" (La aurora, PNY)—strutture, queste, che non potevano non lasciare impronte precise sulla scrittura di un poeta disponibile a sollecitazioni innovative. $\grave{E}$ in rapporto alla figurazione lorchiana di un paesaggio denso di dolore e di violenza che prende radice in Bodini la coscienza di una condizione storico-esistenziale legata alla sua terra, condizione di assoggettazione e sopraffazioni che può essere pienamente evidenziata solo attraverso la metafora del sangue. Meno frequenti, in verità, e contenute, rispetto a Lorca, le immagini violente; alcune comunque si distinguono proprio per il rapporto effettivo con moduli lorchiani: "A coltellate, a tagli viene avanti" (La Brindisina, DL); "Sei un grande pesce senza testa" (Xanti-Yaca, DL); "Rientra decapitato / un carrettiere dalle cave" $(5, F T)$, questi ultimi in sintonia con il "marinero recién degollado" della Navidad en el Hudson (PNY) e immagini affini quali "degolladas voces" (Nacimiento de Cristo, PNY); "un ritmo sin cabeza" (El paso de la siguiriya, PCJ); e la "oración decapitada" del Martirio de Santa Olalla nel Romancero gitano. Tra i versi di Bodini è difficile rinvenire un esempio piú icastico di spictato realismo del seguente: "Lingue penzolanti / di tori squartati / e a testa giú" (Dov'è l'uomo? DL) che rende manifesta la matrice iberica anche nel richiamo al nocturno del hueco (PNY): "En la gran plaza desierta / mugía la bovina cabeza ricićn cortada" senza escludere il lascito montaliano dei "capretti uccisi" in mostra nelle botteghe fiorentine durante la "primavera hitleriana" (La bufera e altro).

Altro dato significativo della fase spagnola di Bodini è costituito da tracciati espressivi che evidenziano la potenzialità dell'aggetti- 
vo quale strumento-chiave in grado di assolvere una funzione non tanto di qualificazione e ampliazione del referente quanto di approfondimento e talvolta di appropriazione connotativa nei confronti del referente. In un processo di transfert analogico, l'aggettivo si trasforma in nucleo semantico centrale dal quale scaturiscono le sfumature tonali e le gradazioni emotive del discorso. Anche qui le suggestioni acquisite dalla frequentazione della poesia di Lorea riecheggiano in termini transitivi di potenziamento metaforico degli oggetti nonché in termini di accentuazioni policromatiche che conferiscono al paesaggio salentino l'identità assoluta di terra meditarranea ove la calce è "regina arsa c concreta" (Finibusterrae, DL) in rapporto di equivalenza con l'Andalusia di Lorca, "tierra tostada" c segnata da "un silencio del cal" (reyerta, $R G$ ).

Va rilevato inoltre l'apporto al registro poctico bodiniano di moduli espressivi lorchiani contraddistinti da una policromia intensa e fortemente suggestiva: "gloria de fuego"; "nidos de ora"; "verde rumor"; "blanco silencio"; "viento amarillo"-colori, dirà Bodini, "che non erano mai stati cosí vivi prima che la poesia di Lorca li cantasse" (Lorca, Tutto il teatro 10. Per la funzione polivalente del colore, Petrucciani 184s.). Colori che riappariranno nelle liriche di Bodini non sotto forma di corrispondenza speculari ma di strutture simboliche che alimentano e potenziano il tracciato poctico anche in funzione di verifica nei riguardi del modello lorchiano. Del resto sussiste in Bodini una disposizione artistica che tende e favorire le attribuzioni nominali ricavate dai colori. È il poeta stesso, nell'osservazione parentetica che chiude la pocsia Con la parola $n u(D L)$ a prenderne atto: "(Chi ha creato il mondo c le sue nere virtú / nulla ha fatto di meglio / dei colori nei campi)." Disposizione colorista che si collega altresí al disegno (anch'esso legato all'esperienza iberica: Macrí, "Pocsia" 76-99) e alle prove narrative dello scrittore salentino. Basti questo escmpio tratto dal racconto /l Sei-Dita: "La pelle del ciclo s'era squarciata $\mathrm{c}$ in tutto il cielo già nero lasciava vedere, in quel punto, delle budella tinte di viola di giallo e verde, e illuminate da una luce spettrale" (La lobbia 93). È in base pertanto alla congiunzione di solle-citazioni esterne e la sensibilità poctica di Bodini che va valutata la dinamicá dell'aggettivazione, generatrice di una straordinaria gamma di colori sempre tesi alla figurazione poliedrica di sentimenti interiori acuiti da inquietudini memoriali. 
In questo riquadro tematico si hanno sintagmi e stilemi cromatici che accentuano la condizione dell'io poetico, condizione segnata da "l'iridiscente pena" (Come un polpo sbattuto, $D L$ ) e dalla "verde noia" di una paesaggio "limpido e disperato" (Quando fu l'ora, $D L$ ). Dello stesso ordine ma improntati a rilevare uno stato d'animo lacerato da trepidanti tensioni, gli "azzurri veleni" (Con questo nome, $L B)$, il "nervoso silenzio" $(9, F T)$, "il delirio d'ali nere nell'aria" (6, FT).

Nell'arco espressivo della Luna dei Borboni e sempre in rapporto alle tecniche di qualificazione, si riscontrano accostamenti suggestivi al poeta granadino. Si pensi ai suoi "corazones de aceite" (Reyerta, $R G$ ) che richiamano, per via di transfert analogico, "gli olivi dal cuore umano" bodiniani (Le mani del Sud, CI; ed è appunto il suddetto modulo nucleare del Lorca che si esteriorizza nei lineamenti femminili di Isobel "dalle braccia d'olio" $(2, L B)$ _immagine che serba il senso tattile di levigata delicatezza della matrice originale nello stesso grado in cui si rivela di netto taglio lorchiano la figurazione di "pazienti donne dagli occhi d'uva" nei versi di Tutto un paese sorge contro un uomo $(D L)$. Significativo altresí, tra le molteplici testimonianze di sinestesia, "la inquietud. . . color de la carne" (Lluvia, LP) che trova raffronto ne "la luce pare / di carne cruda" $(8, L B)$. Altrove il "crepúsculo rojo" di García Lorca è sottoposto a violenta intensificazione nel "tramonto da bestia macellata" $(5, F T)$. Sempre in funzione associativa (sangue-tramonto), l'aggettivo "lordo" trae la sua carica intensiva dal predicato nominale di "Un santo / lordo di sangue nei tramonti" $(11, F T)$. In un'immagine molto simile, "Sanguina Cristo in piaghe / rosso borbonico" (6, $L B$ ), il rosso del sangue si associa a un aggettivo che, avendo precise connotazioni storiche, punta a un rapporto simbiotico, retto dal sentimento della sofferenza, tra la figura di Cristo e la gente del Sud.

Infine va ricordato che tramite l'aggettivazione, e precisamente l'immaginoso interplay policromatico, Bodini ritrova il suo Salento nell'Andalusia di Góngora e García Lorca:

Cordova è una dolce tempesta di bianco verde e nero e in quell'accordo di calce e di limoni e di freschi cancelli trovo il mio Sud ma con piú aperta coscienza con piú aperta coscienza e piú valore.

(Omaggio e Gongora, VDA) 
Tricronomia emblematica il "bianco verde e nero" in cui alla suggestione della tempesta montaliana, mediata dall'ossimoro di radice Ieopardiana, si unisce il verde prediletto da Lorcal ("Verde te quiero verde"; Romance sonambulo, $R G$ ), verde le cui dimensioni surrealistiche (si pensi a "mañana verde"; "ciclo verde"; "verde viento") costituiscono, come sostiene Correal (52), il tessuto mitico del paesaggio andaluso. Del tutto legittimal pertanto risulta l'allusione di Macrí a un processo di "metaforizzazione e sublimazione ispanica" (Introduzione 29) attraverso il quale assume la sua fisionomia storica e i suoi connotati esistenziali il salento mitico di Bodini dal bianco abbagliante e indolente delle sue case di calce. Tramite lo stesso processo, di matrice surrealista iberica $\mathrm{e}$ in gran parte lorchiana, prende forma il "canto nero" (Voli basso, $D L)$ del "Sud nero" (6, $L B)$ ove gli uomini portano "camicie silenziose" ed escono al sole "come numeri / dalla faccia d'un dado" $(1, F T)$ mentre "donne pennute assaggiano il brodo" (Cocumula, $L B$ ) o "allattano i figli sulle porte" (Nella penisola salentina, DL).

Le sostanziali diversità tonali, idcologiche e di strutturazione ritmico-lessicale che intercorrono tra La luna dei Borboni e Mctamor (1967) comportano anche un distanziamento dalla poesia di Lorca, almeno nei termini di cui si è venuto discorrendo. Metamor e le raccolte inedite in vita, Zeta e La Civiltà industriale o poesie ovali, segnano, in un arco di otto anni, l'ultima svolta nella pocsia bodiniana (riscontrabile del resto anche nel Lorca di Poeta en Nueva York), svolta che evidenzia anzitutto il superamento della raffigurazione mitico-esistenziale del Salento, cui è connessa la presenza del pocta andaluso. Le ultime liriche di Bodini risultano espressione di una stagione poetica autunnale, distinta da maggior raccoglimento intellettuale c dalla ricerca di problematiche di piú ampio respiro: il male della civiltà delle macchine, il degrado della cultura nonché "i terrori del vento" (Conosco appena le mani, $M$ ) dell'cra nucleare ravvisabili nella visione apocalittica di un pacsaggio urbano devasiato e deserto ove "statue coi denti strapperanno l'erba / venuta alta, fra i binari del tram" (Finiremo cosí. Inorriditi equinozi, IN).

Il pocta, liberato dalla "guarnigione" del passato legata alla sua terra, si porta all'orizzonte nebuloso della contemporaneità e ne assapora i suoi veleni, che sono anche la nevrosi, la nafta "nell'acqua del marc" (Rapporto del consumo industriale, $\mathrm{Cl}$ ) nonché la doloro- 
sa constatazione che "dov'erano anfiteatri d'uve dizionari d'ombre / si alzano nidi di plastica di cemento di calcoli di gittata" (Ibid.). Ricerca dell'io storico nel passato e affinità con "quegli avi sepolti per tanti secoli / con un profilo come il mio" (Tutto un paese . . DL): tale il contrassegno de La luna dei Borboni; estraniamento dal presente e rifiuto categorico della sua civiltà, distintivo esistenziale di Metamor. Il dissidio interiore, di ordine spirituale nonché metafisico, si articola sul versante di un'introspezionc ossessiva, spietata, lacerante che nei momenti di maggiore sgomento sfocia in "gridi" di straziante desolazione. Il rifiuto del presente-della condizione sociale e personale- c̀ sostenuto da un negativismo assoluto, inesorabile: "Non so"; "Non posso"; "Non si saprà." Oppure: "L'edera mi dicc: non sarai / mai edera. E il vento: non sarai vento" (Canzone semplice dell'esser se stessi, $M$ ). Anche quando l'avvio sintattico si offre in forma construens, "Occorre fare tutto il possibile" (Innesto 13, M), la struttura interna è corrosa da una negatività insistente: "perché le cose non si muovano non spariscano non appena ne distogliamo lo sguardo" (Ibid.; mio il corsivo).

Il rapporto dello scrittore con il microcosmo salentino andaluso, fatto di oggetti "utili” ed "essenziali" (sono termini di Bodini) che ne La luna si è velato di segni arcani e rigencratori dell'io poctico, questo rapporto viene ridotto dall'impenetrabilità degli oggetti che si rifiutano, per cosí dire, di rendersi partecipi del processo creativo:

Dice lo specchio:

come vuoi esser specchio

se non sai dare altro che la tua immagine?

Dicono le cose: cerca d'esser te stesso

senza di noi.

Risparmiaci il tuo amore.

(Canzone semplice dell'esser se stessi, $M$ )

Col venir meno della compenetrazione, la poesia si rivela incapace di acquisire valore transitivo e pertanto si ripiega su se stessa: Poesia triste alla poesia $(Z)$, indugia, si sottomette e tentativi di non-sense: "Credevo che lei credesse e lei credeva / che io credessi. ..." (Credevo che credesse, $Z$ ), di intransività che sfiora il contradditorio: "Vedi la luna rider della luna" (Per un volo nei pressi della luna, CI).

Lontani ormai i ricordi accesi e pregni di vitalità legati al soggior- 
no iberico ("Non chiamatemi ... piú gitano"; $A P^{\prime} 3$ ): pochi e sbiaditi i richiami alla poesia di Lorca, sostituita da altre voci (Mallarmé, Rimbaud, Baudelaire) nonché dal lamentoso Ubi sunt? di stampo villoniano:

\author{
Ah, dove sono le acute presenze \\ del passato, le sue calde forme, \\ la cera su cui incidevano \\ i mici sentinenti?
}

(Conosco appena le mani, M)

In effetti, nell'ultima poesia di Bodini si assiste alla dissoluzione di quella costellazione di immagini vitali ed emblematiche di matrice iberica che costituisce il contrassegno de La luna dei Borboni. In Metamor il paesaggio salentino risulta privo di senso, "insensata pianura" ove l'ombra unita del carro e del cavallo $/ . .$. c ù un solo oggetto gotico / allungato nel sole, senza moto" ( L'ombra unita . . . $I N)$. Figure e oggetti familiari quali appunto il carro c il cavallo, privi dell’intensità lirica originaria, si integrano a un nuovo registro simbolico. Si affacciano alla mente del poeta con connotati diversi e in qualità di immagini del Sud, presente sí ma nella memoria ("hai fatto bene, dice, a non parlarmi del Sud"), immagini che ispirano sentimenti ambigui, di cmpatia per "le capre [del Sud] per metà divorate dallo Stato," c di compiaciuto distacco da un ambiente in cui dominano "l'accusare e l'accusarsi senza pietà" (Le mani del Sud, $C I)$. Assenza e distacco riscontrabili anche nel verso "Fingo d'un carretticre il canto crrante" del poema Scrivo senza mangiare (IN) ove il lascito leopardiano, in funzione antitetica alle suddette sollecitazioni lorchiane, si unisce, nello stesso contesto, a un distico che vanifica senza esitazione la forza del referente simbolico: "Scrivo poesic alla luna, / e chi è la luna?"

L'estrancità nci confronti dell'immagine paradigmatica della luna segna la svalutazione dell'esercizio poetico come ricerca gnoscologica-esistenziale e porta necessariamente, come rileva Chiappini, alla "dissoluzione dell'io poetico vitale" (110) che è quanto dire a un esistenza che ha "il colore della morte" (Se la vita è il colore della morte, $I N)$. Ne risulta un tracciato poctico distinto in gran parte da "immagini del nulla" (Autumno, CI), tracciato in cui il fecondo simbolo lorchiano della luna si rivela ormai estraneo: "Il poeta passeggia . . fra lune altrui” (La passeggiata del poeta, $Z$ ), è ridotto a segno amor- 
fo: "La luna non aveva capo né coda" (Era sicuramente, Z), laido piú che sinistro: "luna calva e grigia" (Per un volo nei pressi della luna, $C I)$. È il simbolo declassato della "luna lumaca" che appare in Sogno $(C I)$ e si dissolve nella poesia Con la parola nu (DL) ove la sillaba $n u$, già radice integrante di "nudità, nulla, nuvola," riaffiora in un interplay associativo di scambio sillabico (luna: nulla) che tende effettivamente ad annullare il valore fonosimbolico del lessema:

Oh se il nulla non fosse solo il nulla ma nuvolaglia polvere poltiglia

nella luna

senza colore

senza nulla. (Ostaggio, $Z$ )

In effetti gli oggetti, privi ormai della loro mediazione generatrice c salvifica, vengono ridimensionati alla luce di un realismo spictato che può dirsi il segno lapidario dell'ultima stagione poctica di Bodini: "Ritrovano le cose nel sonno umano / il silenzio ch'c̀ la loro forma” (Estate. I grandi piatti, VDA).

\section{Syracuse University}

\section{NOTE}

1 Il volume comprende gli atti dei tre convegni di studi su Bodini tenuti, nel dicembre 1980, a Roma, Lecce e Bari. Contributo di notevole valore per la mole del volume (839 pagine), la presenza di studiosi quali Cambon, Dell'Aquila, Leone di Castris, Macrí, Marti, Petrocchi, e Sansone ma soprattutto per il vasto respiro critico degli interventi, suddivisi in quattro sezioni: poesia; prosa e critica; ispanismo e impegno civile; testimonianze.

2 Cf. Omaggio a Bodini ove il contatto del pocta con la Spagna è segnalato da Mario Boselli, Giorgio Caproni, Walter Pedullà e altri come dato degno di ulteriori approfondimenti. Fa eccezione il saggio di Donato Valli (296-342) che a 315 e 319 mette in evidenza, in forma concisa ma molto acuta, il rapporto tra Lorca e Bodini, segnato da "un'atmosfera identica di schictto timbro meridionale."

3 Tale lo stato d'animo di Bodini nei confronti dell'ermetismo ravvisabile nel racconto autobiografico, Firenze, per cui si veda Macrí, Introduzione 15-16 anche per ulteriori riscontri bibliografici.

4 Diamo le sigle delle raccolte poetiche cui risalgono i riferimenti testuali: $F T$ = Foglie di labacco (1945-47); $L B=L a$ luna dei Borboni (1950-51); $D L=$ Dopo la luna (1952-55); VDA = Via de Angelis (1956-60); $M=$ Metamor 
(1962-66). Dei versi inediti, si segnalano: $I N=$ Inedill $(1954-61) ; Z=Z e^{\prime} a$ (1962-69); Cl = Civiltì indestriale (1960-70). Del terzo gruppo, seguendo la sistemazione di Macri ("Appunti di poesie residue e sparse") ma con suddivisione cronologica di riferihilità al periodo spagnolo, segnaliamo: $A P I=$ Appunti di poesie, 1939-46; AP2: 1946-60; AP3: 1960-70.

5 Sullo stesso periodico apparvero, dal 1951 al 1954, ben 24 articoli di interesse spagnolo, frutto del soggiorno madrileno dell'autore. Si veda Giannone, in particolare 372-93.

6 Per ulteriori segnalazioni di scritti critici sull'opera di Bodini, e in particolare per il contributo ragguardevole di Macrí e Valli, si veda la Bibliografia di Macrí, in Bodini, Tutte le poesie, 72-83.

7 Indichiamo le sigle dei testi lorchiani che seguono le nostre segnalazioni: $L P=L i b r o$ de poemas (1921); $P C J=$ Poema del cante jondo (1921); $R G=$ Romancero gitano (1924-27); PN = P'oeta en Nueva York (1929-30); LPI = Llanto por Ignacio Sánchez Mejias (1935); PS = Poemas sueltos.

8 Sull'intensa attività di Bodini traduttore e studioso della letteratura spagnola (di Lorca tradusse il teatro e un gruppo di poesie integrate ai Poeti surrealisti spagnoli), si vedano Tavani, 287-308, e Macrí, "V. Bodini ispanista” 625-80. 